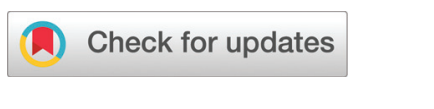

Cite this: Nanoscale, 2020, 12, 16339

\section{Transport of artificial virus-like nanocarriers through intestinal monolayers via microfold cells $\uparrow$}

\author{
Tianjian Tong,,$^{\mathrm{a}}$ Yijun Qi, $t^{\mathrm{b}}$ Luke D. Bussiere, (D) ${ }^{\mathrm{c}, \mathrm{d}}$ Michael Wannemuehler, ${ }^{\mathrm{c}}$ \\ Cathy L. Miller, ${ }^{c, d}$ Qun Wang (D) *b and Chenxu Yu (D)*a
}

Received 11th May 2020,

Accepted 6th July 2020

DOI: $10.1039 /$ dOnr03680c

rsc.li/nanoscale

\begin{abstract}
Compared with subcutaneous or intramuscular routes for vaccination, vaccine delivery via the gastrointestinal mucosa has tremendous potential as it is easy to administer and pain-free. Robust immune responses can be triggered successfully once the vaccine carrying an antigen reaches the mucosal associated lymphoid sites (e.g., Peyer's patches). However, the absence of an efficient delivery method has always been an issue for successful oral vaccine development. In our study, inspired by mammalian orthoreovirus (MRV) transport into the gut mucosal lymphoid tissue via Microfold cells (M cells), artificial virus-like nanocarriers (AVNs), consisting of gold nanocages functionalized with the $\sigma 1$ protein from mammalian reovirus (MRV), were tested as an effective oral vaccine delivery vehicle targeting $M$ cells. AVNs were shown to have a significantly higher transport compared to other experimental groups across mouse organoid monolayers containing $M$ cells. These findings suggest that AVNs have the potential to be an $\mathrm{M}$ cell-specific oral vaccine/drug delivery vehicle.
\end{abstract}

\section{Introduction}

Oral vaccination targeting the gastrointestinal (GI) mucosa layer is considered more patient-friendly than subcutaneous injection or intravenous injection with regard to induction of a protective immune response. ${ }^{1}$ GI mucosal delivery of vaccines has additional practical advantages over vaccination by intravenous injection or subcutaneous injection, including both cost efficiency and increased safety. ${ }^{2}$ While progress has been made substantially with the increased stability of and immune activation by mucosal vaccines, practical and effective oral vaccine delivery systems targeting the GI mucosa remain to be developed due to poor understanding of specific and nonspecific factors determining the delivery, targeting, recognition and transport of vaccines/vaccine carriers across the GI mucosal epithelia. In this article, we aimed to validate the development of an artificial virus-like nanocarrier which specifically targets $\mathrm{M}$ cells for transcytosis, providing a more effective strategy to deliver oral vaccines into mucosal associ-

\footnotetext{
${ }^{a}$ Department of Agricultural Biosystem and Engineering, Iowa State University, Ames, Iowa, USA. E-mail: chenxuyu@iastate.edu

${ }^{b}$ Department of Chemical and Biological Engineering, Iowa State University, Ames, Iowa, USA.E-mail: qunwang@iastate.edu

${ }^{c}$ Department of Veterinary Microbiology and Preventive Medicine, College of Veterinary Medicine, Iowa State University, Ames, Iowa, USA

${ }^{d}$ Interdepartmental Microbiology Program, Iowa State University, Ames, Iowa, USA

$\dagger$ Electronic supplementary information (ESI) available. See DOI: 10.1039/ d0nr03680c

\$These two authors contributed equally to this article.
}

ated lymphoid tissues (e.g., Peyer's patches) for enhanced vaccine efficacy.

Type 1 Lang mammalian reovirus (MRV) is an $85 \mathrm{~nm}$ diameter, non-enveloped virus composed of 5 structural proteins $(\lambda 1, \lambda 2, \lambda 3, \sigma 2$, and $\mu 2)$, forming a core particle that encloses the virus genome, surrounded by 3 outer capsid proteins $(\sigma 1$, $\mu 1$, and $\sigma 3$ ) that play critical roles in virus cell entry. ${ }^{3}$ Following oral inoculation, T1L MRV accesses mucosal associated lymphoid tissue (MALT) by transcytosis through M cells, which are specialized cells located at the follicle-associated epithelium (FAE) of Peyer's patches. ${ }^{4,5} \mathrm{M}$ cells continuously sample for foreign antigens within the lumen by endocytosing antigens suspended in the fluid at the apical surface of the cell. These antigens are then transcytosed from the apical surface to the basolateral surface. Since there is a thick mucus layer on the apical surface of the enterocytes, viruses often exploit $\mathrm{M}$ cell sampling to gain access to the basolateral side of the enterocytes where infection can proceed. In the case of MRV, the MRV outer capsid protein, $\sigma 1$, adheres to $\alpha 2-3$-linked sialic acids on the $\mathrm{M}$ cell apical surface, and then the T1L MRV is transcytosed across the M cell to the MALT where it can initiate infection of enterocytes on the basolateral surface of these cells. ${ }^{6}$

Taking inspiration from the natural pathway of MRV transcytosis across $\mathrm{M}$ cells (Fig. 1a), we hypothesized that artificial virus-like nanocarriers (AVNs), composed of T1L $\sigma 1$ conjugated gold nanocages, would be able to effectively transcytose through $\mathrm{M}$ cells. Hence, these AVNs can serve as effective transport vehicles for oral vaccines. AVN construction required 
a

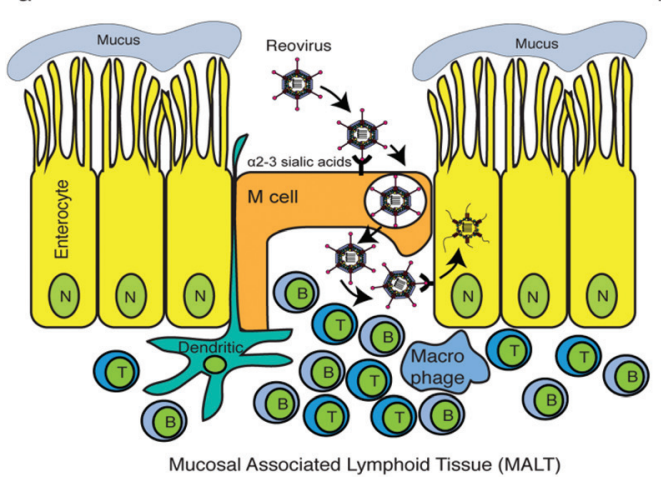

b

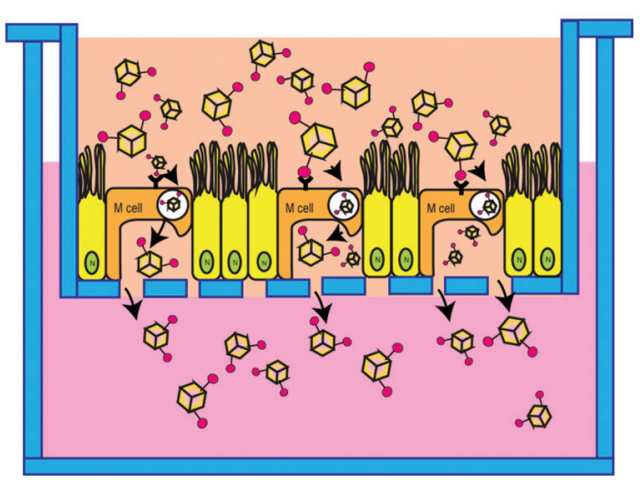

Fig. 1 Scheme: T1L $\sigma 1$ functionalized gold nanocages exploit the M cell pathway. (a) Model of MRV infection of enteric tissue. (b) Experimental design to test the transport of $\sigma 1$ functionalized AVNs through $M$ cells incorporated to intestinal organoid monolayers.

purification of $\sigma 1$, preparation of gold nanocages, and linkage of $\sigma 1$ to the nanocage surface in an appropriate conformation where the head domain (C-terminal portion) $)^{7}$ of $\sigma 1$ can interact with $\alpha 2-3$ sialic acid located on the $\mathrm{M}$ cell apical surface. ${ }^{6}$

With the nanocarriers being surface-functionalized with T1L $\sigma 1$, conventional 2-D spherical nanoparticles were not good candidates as the outer surface would no longer be available for payload loading. In this study, we chose gold nanocages (i.e., hollow nanocubes) as our base for nanocarrier development mainly due to their easiness to fabricate and their internal loading capacity. The nanocages had holes to allow loading/ unloading of payloads. A coating could be added to cover the outer surface to further protect the payloads sealed inside for oral vaccines to survive the harsh gastric environment.

To test our hypothesis, a simple and effective testing platform was developed using $\mathrm{M}$ cell containing intestinal monolayers derived from mouse intestinal organoids in which $\mathrm{M}$ cell differentiation was induced using recombinant RANK ligand (RankL). ${ }^{8}$ Compared with a monolayer of Caco-2 cells, the organoid monolayer contains various epithelial cell types, such as goblet cells, enteroendocrine cells, enterocytes and Paneth cells. ${ }^{9}$ The development of an ex vivo intestinal mucosal system composed of a continuously expanding, selforganizing structure, reminiscent of a normal intestinal epithelium, provides an alternative that bridges the gap between cell lines and in vivo animal models for the study of oral vaccines. Based on previous studies, ${ }^{10}$ the organoid generated monolayers are polarized as evidenced by the fact that they maintain apical and basally oriented membranes, which are a critical component of our model as MRV adheres to the apical membrane of $\mathrm{M}$ cells in vivo. ${ }^{11}$ Additionally, from previous studies, $\mathrm{M}$ cells can be induced in both $2 \mathrm{D}$ and $3 \mathrm{D}$ organoid systems. ${ }^{12}$ Our experimental setup used Transwell plates containing an $\mathrm{M}$ cell embedded organoid monolayer mimicking an intestinal mucosal layer on the top inserts, with the bottom compartment mimicking a MALT site (Fig. 1b). Using this system, we examined the capacity of AVNs to exploit M cellmediated uptake through interaction of $\sigma 1$-conjugated AVNs with $\alpha 2-3$ sialic acids followed by transcytosis through $\mathrm{M}$ cells.

\section{Results and discussion}

\section{Artificial virus-like nanocarriers: gold nanocages and MRV $\sigma 1$}

Gold nanoparticles are widely used in the drug/vaccine delivery field. ${ }^{13-18}$ Gold nanocages (GNCs) are 3D structures with a hollow interior which allows a substantially increased loading capacity for payload (e.g., drugs or immunogens) when compared with $2 \mathrm{D}$ delivery vehicles. GNCs were produced using a two-step galvanic replacement reaction. ${ }^{15,16}$ In the first step, silver nanocubes were fabricated as sacrificial templates, and in the second step, a galvanic replacement reaction was utilized to replace silver with gold. 3D hollow gold nanocages were obtained from this reaction with an average size of $40 \mathrm{~nm}$ (Fig. 2a). GNCs utilized throughout our study had an absorption peak at around $760 \mathrm{~nm}$ (Fig. 2b). MRV $\sigma 1$ was harvested from purified MRV as previously described. ${ }^{19-21}$ Presence of $\sigma 1$ protein is confirmed by western blot using the T1L virion antisera which recognize $\sigma 1$ along with other capsid proteins. In Fig. 2c, our western blot analysis revealed one band at $50 \mathrm{kDa}$ representing $\sigma 1$ protein (right lane). No band was detected in the filtered suspension (middle), indicating that there was no unconjugated $\sigma 1$ protein present, while in the post-purification pellet (left), all MRV structural proteins were revealed. EDC/ NHS mediated conjugation was utilized to link $\sigma 1$ proteins onto $\mathrm{GNCs}^{22,23}$ to produce AVNs. Conjugation success was confirmed by incubation of AVNs as well as GNCs without $\sigma 1$ conjugation (i.e., control) with gold nanospheres (GNSs) conjugated with a $\sigma 1$ specific monoclonal antibody (5C6). While the control maintained absorption peaks representing free-floating GNCs $(760 \mathrm{~nm})$ and GNSs $(550 \mathrm{~nm})$, the (GNC- $\sigma 1)+($ GNS-5C6) group showed no absorption, indicating aggregation via $\sigma 1-5 \mathrm{C} 6$ crosslinking and confirming $\sigma 1$ conjugation to GNCs.

\section{Microfold cell (M cell) incorporated intestinal monolayer preparation}

Intestinal organoids are 3-dimensional structured epithelia that are derived from small intestinal stem cells. ${ }^{9}$ Stem cells were isolated from the small intestine of $\mathrm{C} 3 \mathrm{H} / \mathrm{HeN}$ conventional mice and mixed with Matrigel containing growth medium 
a

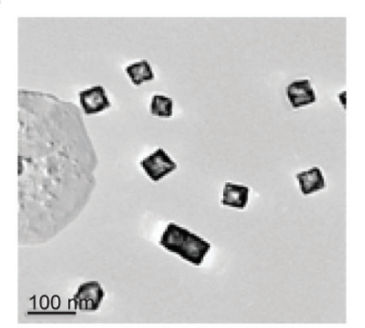

b

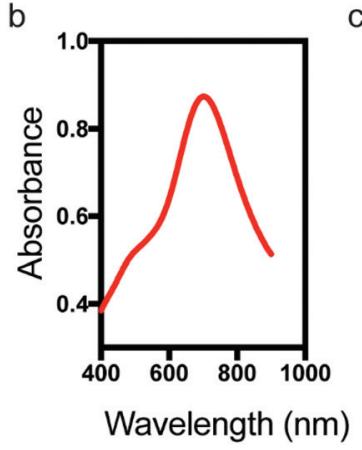

c

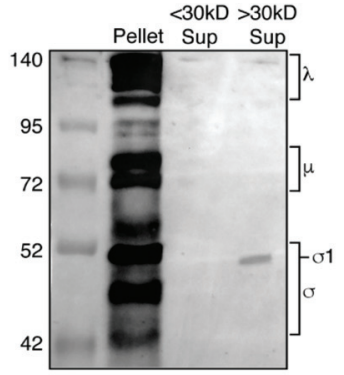

d

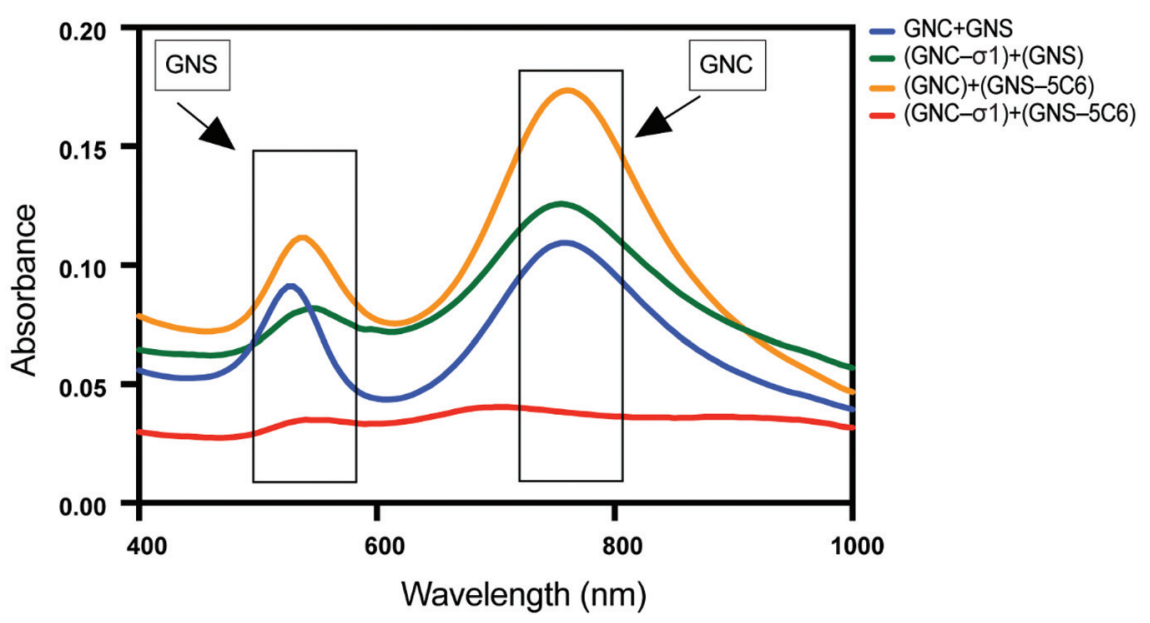

Fig. 2 MRV $\sigma 1$ functionalization of GNCs. (a) TEM (Transmission Electron Microscopy) image of GNCs showing $40 \mathrm{~nm}$ average size. (b) UV (Ultraviolet) absorption of GNCs shows a characteristic peak at $760 \mathrm{~nm}$. (c) Western blot for pellets after last centrifugation, control group and T1L $\sigma 1$ protein $(50 \mathrm{kDa})$. Following purification, T1L $\sigma 1$ protein was concentrated using a $30 \mathrm{kDa}$ filter (right). Filtrated solution (middle) and pellets (left) serve as the control. (d) GNCs conjugated with MRV $\sigma 1$ protein (GNCs $+\sigma 1$ ) or (GNCs) alone, and GNSs conjugated with $\sigma 1$ monoclonal antibody 5 C6 (GNSs $+5 C 6$ ) or not (GNSs) were incubated together and absorption was measured to identify aggregation.

supplemented with growth factors including R-spondin 1, EGF, and Noggin. ${ }^{24}$ Previous studies ${ }^{25-29}$ have demonstrated that adding RankL to the organoid culture medium induced the $\mathbf{M}$ cell appearance. The expression of $\mathbf{M}$ cells was confirmed by expressing $\mathbf{M}$ cell surface protein glycoprotein 2 (GP2) and M-cell-specific molecule annexin V (Anxa5) ${ }^{5}$ on organoids. Additionally, biotin-conjugated lectin UEA-1 (UEA-1) binds specifically to the sites of $\alpha-1,2$ fucosylation on the surfaces of $\mathrm{M}$ cells. ${ }^{30}$ Hence, UEA-I and GP-2 are both specific markers for RankL induced $M$ cells. While UEA- 1 binds specifically to the apical sites of $\alpha-1,2$ fucosylation on the surfaces of $\mathrm{M}$ cells in the epithelial layer, GP-2 binds specifically to the basal site surface of $\mathbf{M}$ cells in the epithelial layer. As shown in Fig. 3a, the fluorescence signals for GP-2 and UEA-1 indicated the distribution of the induced M-cells at both apical sites and basal sites.

Based on these observations, $M$ cells were induced in $3 \mathrm{D}$ structured organoids by a 3-day incubation in the presence of RankL. Similar to prior studies, ${ }^{25-29}$ we found lectin UEA-I binding (shown as red) and $M$ cell marker GP2 expressed (shown as green) in organoids treated with RankL, but not in untreated organoids (Fig. 3a). For further verification, gene expression analysis was performed using RT-qPCR and primers specific for Anxa5 and GP2. Consistent with the expression of $\mathbf{M}$ cell markers seen by immunofluorescence, organoids treated with RankL significantly upregulated the expression of both Anxa5 and GP2 relative to untreated cells (Fig. 3b). Finally, western blot was performed using antibodies specific for annexin $\mathrm{V}$ in which RankL induced organoids showed a specific band with the correct molecular weight that was not present in the untreated organoids (Fig. 3c).

Taken together, these findings strongly suggest that a 3-day incubation with RankL resulted in the induction of $\mathbf{M}$ cells within the organoids. While intact 3D organoids are useful for many experiments, our assay required a monolayer, and it has been demonstrated previously that organoids can be disrupted and plated on tissue culture plates to form a monolayer. ${ }^{10}$ In order to achieve this, we treated organoids for 2 days with RankL, disrupted and re-plated the cells as a monolayer in the presence of RankL for an additional 24 hours on the top compartment of a Transwell tissue culture insert (Fig. 1b) for further experiments. As shown in Fig. 3d, organoid monolayers positively stained for GP2, which was absent in control samples which did not receive RankL, indicating that M cells 
a RankL

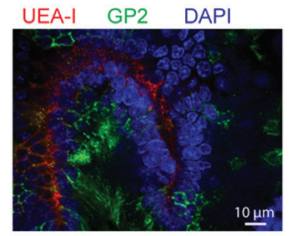

C

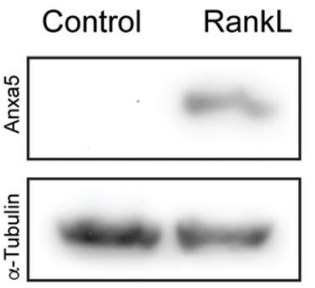

b

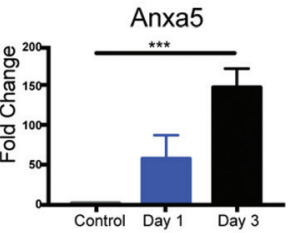

RankL E-CAD GP2 DAPI

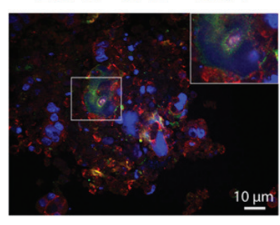

GP2

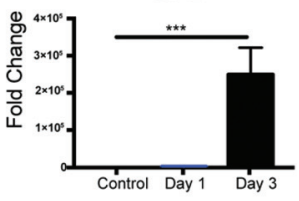

Control
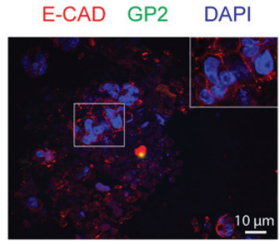

Fig. 3 Detection of microfold cells in 3D mouse organoids and 2D organoid-derived monolayers. (a) Immunofluorescence images of untreated and 3 day RankL-treated 3D organoids with antibodies against $M$ cell marker proteins GP2 (green) and UEA-I lectin (red). Nuclei are stained with DAPI (blue). (b) RT-qPCR analysis for expression of Anxa5- and GP2-specific mRNA isolated from untreated and RankL-treated organoids. (c) Western blot analysis of cell lysates from untreated and RankL-treated organoids using antibodies against Anxa5. (d) Immunofluorescence images of organoid monolayers stained with antibodies against GP2 (green) and E-cadherin (red). Nuclei are stained with DAPI (blue). The boxed region in the merged image was amplified and is shown in the inset.
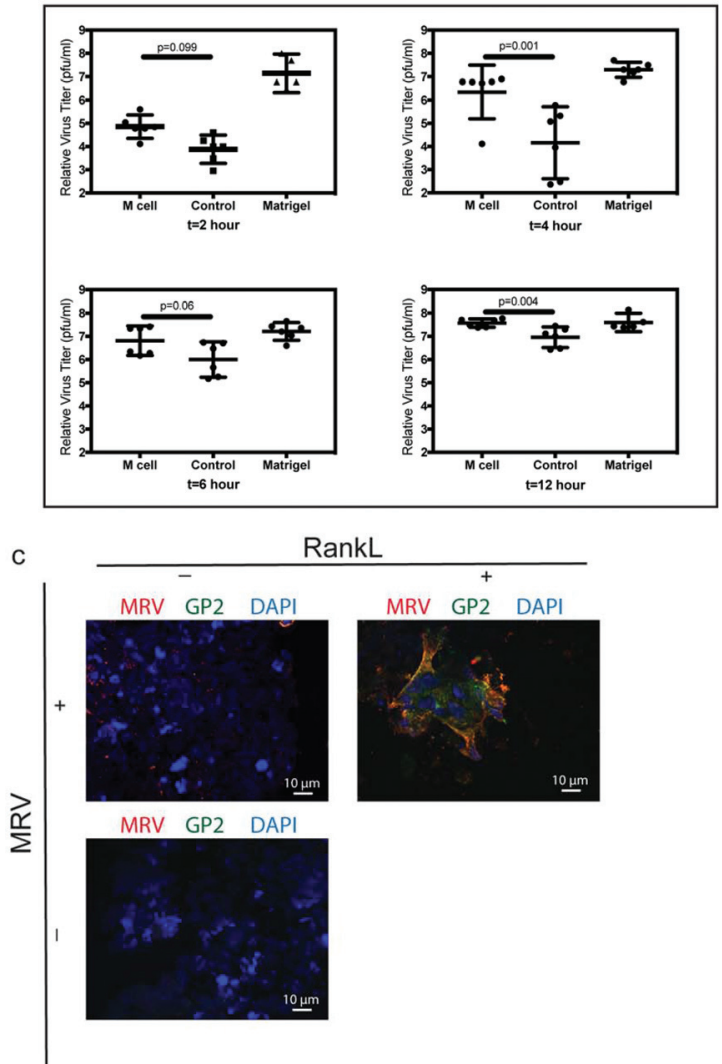

b

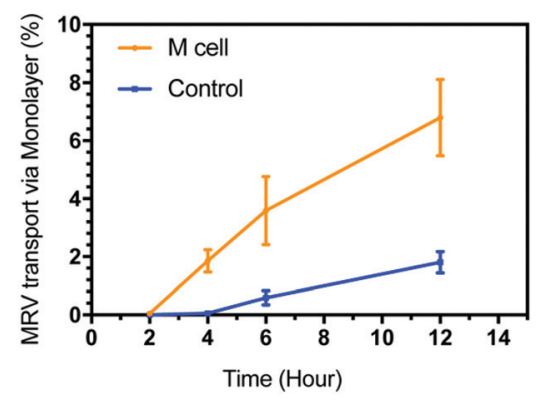

Fig. 4 MRV transport behavior on organoid monolayers. (a) PFU of MRV transport through $M$ cell induced monolayers, monolayers with $M$ cell induction (control) and 1\% Matrigel without monolayers for 2, 4, 6 and 12 hours. $p$ values were calculated and indicated on top of the bar line. (b) MRV transport total at 2, 4, 6, and 12 hours, on monolayers with and without M cell induction. (c) Immunofluorescence images of MRV-infected monolayers treated or untreated (control) with RankL using antibodies against MRV and GP2. 
were present in the monolayer system. Additionally, E-cadherin staining was performed to verify the presence of tight junctions within the monolayers in both control and RankL-treated groups (Fig. 3d). The morphology of the monolayers is further demonstrated by bright field images in ESI Fig. $1 . \dagger$

\section{Transport behavior of mammalian orthoreovirus through organoid monolayers}

As proof of the concept that our organoid monolayer recapitulates MRV transcytosis through M cells, experiments were conducted to measure MRV transport through intestinal organoid monolayers with or without $\mathbf{M}$ cells in the Transwell system (Fig. 1b). Purified MRV was added to untreated or RankLtreated organoid monolayers plated on the top compartment of the Transwell. At 2, 4, 6, and 12 hours, culture medium from the bottom compartment was collected and used for virus plaque assay to examine the amount of virus that passed through the monolayer as a measure of MRV transcytosis through $M$ cells. At each time point, there was an increase in MRV transport into the bottom Transwell in RankL-treated versus control monolayers, with two of the time points showing statistically significant differences. Moreover, when measuring the transport total of MRV across the organoid monolayer, the presence of RankL significantly increased the speed at which the virus moved into the bottom compartment (Fig. 4b) (ESI Note $1 \dagger$ ). To confirm M cell presence and MRV infection in these monolayers, the $4 \mathrm{~h}$ time point was immunostained with antibodies against GP2 and MRV (Fig. 4c). A noticeable overlap of GP2 and MRV staining can be observed in the RankL treated samples, further supporting MRV binding and transport through $\mathbf{M}$ cells in this system. Taken together, these data corroborate previously published data that MRV exploits the $\mathrm{M}$ cell pathway for transport into the MALT and support our hypothesis that this system can be used for measuring $\mathbf{M}$ cell dependent transport of AVNs across the gut mucosa.

\section{Transport of AVNs through organoid monolayers}

Following the production and validation of AVNs and organoid monolayers, we tested our hypothesis that AVNs could be transported across the gut monolayer via $\sigma 1$ binding and transcytosis through $\mathrm{M}$ cells. Using the small molecule dye Rhodamine 6G (R6G) as the payload within the GNCs and AVNs, monolayers with and without $M$ cells were overlaid with R6G (baseline for transport), R6G loaded GNCs, and R6G loaded AVNs. At $0,0.5,1,2,3,4,8,12,24,36$, and 48 hours post-treatment, samples were removed from the lower compartment of Transwells and fluorescence was measured (Fig. 5a). These experiments demonstrated that AVNs display the highest transport across the organoid monolayer containing $\mathbf{M}$ cells among all four experimental groups. Moreover, while there are no differences in total transport between monolayers with and without $M$ cells in the R6G and GNC groups, the addition of $\sigma 1$ to the AVN group resulted in an increase in transport in the presence of $M$ cells, strongly suggesting that the AVN group is using the $\sigma 1: \mathrm{M}$ cell-mediated transcytosis pathway. When examining the first 8 hours, the transcytosis of AVNs across M a

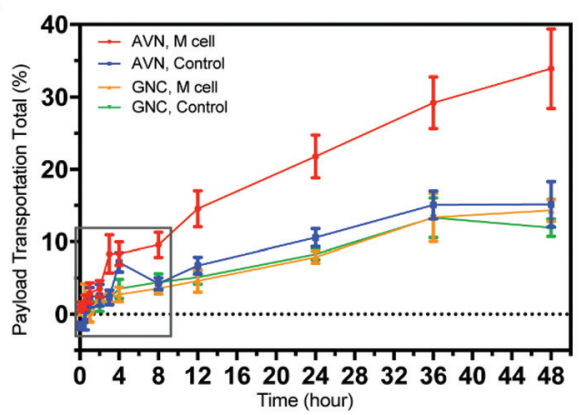

C

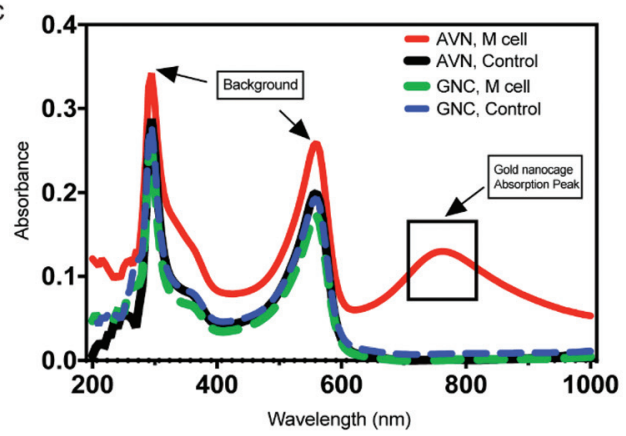

b
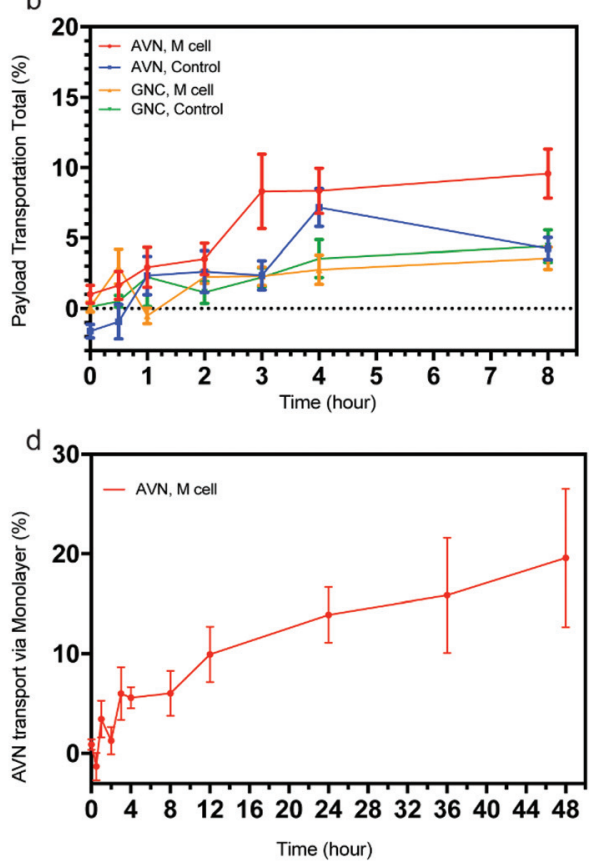

Fig. 5 Transport behavior on the monolayer platform. (a) Payload (R6G) in different vaccine delivery vehicle (AVNs and GNCs) transport across intestinal monolayers, with and without $M$ cell induction over time. (b) First 8 hours in the boxed region of a and (c) UV absorption of each sample at 48 hours. (d) AVN transport total through intestinal monolayers with M cells incorporated. 
cell containing monolayers is distinguished from the other three experimental groups, in which the transport total was higher than for AVNs across monolayers without $\mathbf{M}$ cells (Fig. 5b). As for GNCs, regardless of whether $\mathrm{M}$ cells were present in the monolayers, the transport total was significantly lower than that of the AVNs (ESI Note $2 \dagger$ ). At early time points (hour 1 to 4), AVNs possessed a higher transport total than free R6G (ESI Fig. $2 \dagger$ ). In all four groups, some R6G payload diffused out of the nanocages throughout the experiments, and was detected in the bottom compartment. We suggest that R6G is released from the nanocages in the top compartment and transported through the monolayers as small molecules do. When examining the UV spectra of samples collected from the bottom compartment after 48 hours, only the AVNs on monolayers with $\mathbf{M}$ cells contain a specific gold nanocage absorption peak ( $\sim 60 \mathrm{~nm})$ in the bottom solution (Fig. 5c). This strongly indicates that only the AVNs can transport through the monolayer, and further only in monolayers with $\mathrm{M}$ cell presence, most likely via $\sigma 1$-mediated transcytosis through the $\alpha 2-3$ sialic acid pathway. Gold nanocages otherwise cannot go through the intestinal monolayer, regardless of whether they are T1L $\sigma 1$ functionalized or not. Therefore, both of the two factors, T1L $\sigma 1$ and $\mathrm{M}$ cells, are needed for the transport of AVNs through intestinal organoid monolayers. We performed a quantitative assessment of how many payloads (R6G) inside AVNs transported through monolayer (ESI Note $3 \dagger)$. The overall transport of payloads in AVNs was $20 \%$ at 48 hours (Fig. 5d). These results strongly suggest that AVNs could be quite effective in transporting payloads through $\mathbf{M}$ cells in the gut mucosa, and potentially serve as promising oral vaccine carriers.

\section{Conclusion}

To summarize, artificial virus-like nanocarriers (AVNs) composed of gold nanocages functionalized with MRV receptor binding protein $\sigma 1$ were created which could effectively utilize $\mathrm{M}$ cells for transport across intestinal monolayers, mimicking the MRV transport pathway. Analysis revealed that both MRV $\sigma 1$ functionalization of the AVNs and M cell presence in the monolayer were needed for demonstrable transport across the monolayer. The transport was reasonably efficient with $\sim 20 \%$ of total AVNs transported across the intestinal monolayer within 48 hours. These data suggest that AVNs, carrying an antigen or drug payload, could be used for oral delivery across the gastrointestinal mucosa into mucosal associated lymphoid sites (e.g., Peyer's patches) for enhanced vaccine effectiveness.

\section{Experimental}

\section{Gold nanocage fabrication}

Ethylene glycol (EG) (Sigma-Aldrich) (6 ml) was heated in a $160{ }^{\circ} \mathrm{C}$ oil bath for 1 hour. Polyvinylpyrrolidone (PVP) (SigmaAldrich) (0.07 g) was added into $3.5 \mathrm{ml}$ EG solution, and mixed well. $\mathrm{AgNO}_{3}$ (Sigma-Aldrich) (0.12 g) was added into $2.5 \mathrm{mg}$ EG solution and mixed well. Well mixed $3 \mathrm{mM} \mathrm{NaSO}_{4}$ (Acros) EG solution was made by adding $70 \mu \mathrm{l}$ of sodium sulfide solution to the EG solution in the oil bath. 10 minutes later, $1.5 \mathrm{ml}$ PVP solution and $0.5 \mathrm{ml}$ silver nitrate solution were added into the reaction system and reacted for 10-15 $\mathrm{min}$. At the end of this incubation, $6 \mathrm{ml}$ of acetone were added to cool down the reaction mixture. The silver nanocube solution obtained from the above steps was centrifuged at $1300 \mathrm{rpm}$ for $30 \mathrm{~min}$. The supernatant was removed, and $1 \mathrm{ml}$ of deionized water was added. The nanocubes were then re-suspended in DI water in a sonicator for at least 1 hour. Then the nanocubes were washed twice and collected by centrifugation at $9000 \mathrm{rpm}$, before being re-dispersed in de-ionized water.

PVP (20 mg) was dissolved in $20 \mathrm{ml}$ of deionized water. The PVP solution was then heated to $245{ }^{\circ} \mathrm{C}$ and stirred at 220 rpm. Once the PVP solution started to boil, $200 \mu \mathrm{L}$ of silver nanocubes were added into it. The combined solution was stirred and heated for an additional 10 minutes. $10 \mu \mathrm{L}$ of $\mathrm{HAuCl}_{4}$ (Sigma-Aldrich) was then added in 2-minute increments until the desired concentration was attained (determined by solution color). Further verification of the concentration was done using UV-Vis spectrometry. The solution was then boiled down to $1 \mathrm{ml}$ to concentrate the gold nanocages and $\mathrm{NaCl}$ was added in excess into the solution until saturation. $^{17,18}$ The GNC solution was centrifuged for 30 minutes at $2000 \mathrm{~g}$ to form a pellet. The supernatant was removed, and $1 \mathrm{~mL}$ of distilled water was added. The solution was sonicated for 1 hour to re-disperse the pellet back into a stable solution. Then the nanocages were centrifuged at $9000 \mathrm{~g}$ for 10 minutes and re-dispersed in a sonicator for 1 hour. This last step was repeated one more time. The washed gold nanocages were stored at $4{ }^{\circ} \mathrm{C}$.

\section{T1L $\sigma 1$ isolation and purification}

L929 mouse fibroblast cells were maintained in Joklik modified minimum essential medium (Sigma-Aldrich) supplemented with $2 \%$ fetal bovine serum, $2 \%$ bovine calf serum (HyClone), $2 \mathrm{mM}$ L-glutamine (Mediatech), and penicillin (100 IU ml $\left.{ }^{-1}\right)$-streptomycin $\left(100 \mu \mathrm{g} \mathrm{ml}^{-1}\right)$ solution. MRV strain type 1 Lang (T1L) was propagated in spinner adapted L929 cells, and the cells were harvested by centrifugation at $3000 \mathrm{~g}$ for $10 \mathrm{~min}$, resuspended in $\mathrm{HO}$ buffer $(250 \mathrm{mM} \mathrm{NaCl}, 10 \mathrm{mM}$ Tris, $\mathrm{pH}$ 7.4), and frozen at $-80{ }^{\circ} \mathrm{C} .{ }^{19}$ Virus was purified as described $^{19}$ with the substitution of Vertrel® XF (DuPont) in place of Freon. ${ }^{21}$ Purified MRV was then digested with $\alpha$-chymotrypsin and purified to produce ISVPs, ${ }^{20}$ and then heated to $52{ }^{\circ} \mathrm{C}$ for $30 \mathrm{~min}$ to release $\sigma 1 .^{19}$ The ISVPs were pelleted at $35000 \mathrm{rpm}$ and the supernatant containing free $\sigma 1$ was concentrated in a Centricon-30 microconcentration unit (Amicon Corp.) as described. ${ }^{19}$

\section{Gold nanocage functionalization}

GNC functionalization was achieved using a (1-ethyl-3-(3-dimethylaminopropyl) carbodiimide hydrochloride) (EDC) (Thermo scientific)/ $N$-hydroxysulfosuccinimide (NHS) (Sigma- 
Aldrich) procedure. 4-Aminothiophenol was linked onto GNCs by shaking overnight. The solution was centrifuged at $9000 \mathrm{~g}$ for 10 minutes, then DI-water was added, and GNCs were redispersed in a sonicator for 30 minutes to remove excess 4-aminothiophenol. Freshly made EDS/NHS solution was added to GNCs for $30 \mathrm{~min}$ at room temperature. MRV T1L $\sigma 1$ protein aqueous solution was added and incubated for 2 hours at room temperature, and the reaction was stopped with $1 \mathrm{ml}$ PBS with Tween-20 (PBST). Finally, the solution was centrifuged at $6500 \mathrm{~g}$ for $30 \mathrm{~min}$, the supernatant was removed, and $2 \mathrm{ml}$ of deionized water were added to re-disperse the nanocages. $^{22,23}$

\section{High-resolution immunofluorescence microscopy}

Organoids were grown in 4-well chamber slides and were fixed with $4 \%$ paraformaldehyde for 1 hour at room temperature. Sections were permeabilized with $0.5 \%$ TritonX buffer $(250 \mu \mathrm{L}$ Triton-X100 in $50 \mathrm{~mL}$ PBS) for 30 minutes, and after 3 PBS washes, 1\% PBSA was used for making primary antibody solution and then stained overnight at $4{ }^{\circ} \mathrm{C}$. Then, at day 2 , secondary antibodies were added and incubated at room temperature for 2 hours. After 3 times PBS washes, 4',6-diamidino-2-phenylindole (DAPI) (EMD Millipore) was used to stain nuclei and stick cells under cover slips. After drying the slides for 1 day in the dark, they were ready to use for observation under a microscope. Fluorescence staining images were acquired with an Olympus®IX81 laser scanning confocal microscope using a $100 \times$ oil-immersion objective.

For staining with UEA-1, Lectin Ulex europaeus (biotinylated UEA-1, Sigma) and rabbit anti-biotin (Rockland) were used with a suggested concentration of $10 \mu \mathrm{g} \mathrm{ml}{ }^{-1}$. For secondary antibody, goat anti-rabbit antibody - Texas Red X (Invitrogen) were used at a $1: 250$ dilution. For staining GP2, primary antibody Rat anti-GP2 (MBL International Corporation) was used with a concentration of $2.5 \mu \mathrm{g} \mathrm{ml} \mathrm{m}^{-1}$, and secondary antibody goat anti-rat FITC (ThermoFisher) was used at a 1:250 dilution. For staining E-cadherin, primary antibody rabbit anti-E-cadherin (Invitrogen) was used at a $1: 250$ dilution, followed by a secondary antibody donkey anti-rabbit IgG H\&L (Alexa Fluor® 594) (Abcam) at a 1:250 dilution. For MRV staining, rabbit $\mathrm{T} 1 \mathrm{~L}$ anti-virion antibody, mouse anti- $\sigma 35 \mathrm{C} 3$, mouse anti- $\sigma 310 \mathrm{C} 1$ and mouse anti- $\mu 14 \mathrm{~A} 3$ were used at a 1:250 dilution. ${ }^{31,32}$ The following day, secondary antibody donkey anti-mouse Alexa Fluor® 594 (Invitrogen) and donkey anti-rabbit IgG H\&L (Alexa Fluor® 594) at a 1:250 dilution were used.

\section{Quantitative RT-PCR for verifying microfold cell development}

Matrigel was removed from organoids by treating them with $1000 \mu \mathrm{l}$ of Cell Recovery Solution (Corning Life Sciences) with shaking for $1 \mathrm{~h}$ at $4{ }^{\circ} \mathrm{C}$, followed by two PBS washes. RNA was extracted by using an RNeasy Mini Kit (Qiagen) following the manufacturer's procedure. Concentration and purity of the extracted RNA were checked by using a NanoDrop spectrophotometer. Three primer pairs were purchased from the DNA facility, Iowa State University, Ames, IA: Anxa5 (F: 5'-
ATCCTGAACCTGTTGACATCCC-3'; R: 5'-AGTCGTGAGGGCTT CATCATA-3'), GP2 (F: 5'-CTGCTACCTCGAAGGGGACT-3'; R: 5'-CATTGCCAGAGGGAAGAACT-3') and a primer for the housekeeping gene Gapdh (F: 5'-TTCACCACCATGGAGAAGGC-3'; R: 5'-GGCATGGACTGTGGTCATGA-3'). A Power SYBR ${ }^{\mathrm{TM}}$ Green RNA-to-CT ${ }^{\mathrm{TM}}$ 1-Step Kit was used. Gene expression levels were normalized against that of the housekeeping genes.

\section{Western blot for verifying the presence of $\mathrm{M}$ cell related proteins and $\sigma 1$ protein}

Matrigel was removed from organoids by treating them with $1000 \mu \mathrm{l}$ of Cell Recovery Solution (Corning Life Sciences) with shaking for $1 \mathrm{~h}$ at $4{ }^{\circ} \mathrm{C}$, followed by PBS washes. For $\sigma 1$ identification, MRV was collected in protein loading buffer. Cells or MRV was denatured by heating for 5 to $10 \mathrm{~min}$ at $100{ }^{\circ} \mathrm{C}$. Proteins were separated on a $4 \%$ Tris-HCl SDS-PAGE gel. Gels were transferred to a nitrocellulose membrane and western blotting was performed with rabbit anti-Anxa5 antibody (Abcam) at a dilution of $1: 500$ and anti- $\alpha$-tubulin monoclonal mouse antibody (ThermoFisher Scientific) at a dilution of $1: 1000$. For $\sigma 1$ protein, western blotting was performed with rabbit T1L virion antibody at a dilution of $1: 1000$. Blots were washed $3 \times$ in Tris-buffered saline $(20 \mathrm{mM}$ Tris, $137 \mathrm{mM} \mathrm{NaCl}$ [pH 7.6]) with $0.25 \%$ Tween 20 (TBST) buffer, and then incubated with secondary goat anti-rabbit IgG (BioRad, 170-6518) at a dilution of $1: 2500$. After three additional washes, membranes were subjected to PhosphaGLO AP (SeraCare) to image detected proteins. ${ }^{3}$

\section{Minigut monolayer development}

Murine organoids were cultured for 3 days using well established methods. ${ }^{9}$ At day 3, for $\mathrm{M}$ cell differentiation, $200 \mathrm{ng}$ $\mathrm{ml}^{-1}$ RankL was added and incubated for an additional 2 days. Resulting 3D structured organoids were disrupted and pipetted into buffer containing $0.5 \mathrm{mM}$ EDTA, and centrifuged at $200 \mathrm{~g}$ at $4{ }^{\circ} \mathrm{C}$ for $5 \mathrm{~min}$. $500 \mu \mathrm{l}$ of $0.05 \%$ trypsin $/ 0.5 \mathrm{mM}$ EDTA was added to the pellet for dissociation. After incubation for $4 \mathrm{~min}$ at $37{ }^{\circ} \mathrm{C}, 1 \mathrm{ml} \mathrm{DMEM} / \mathrm{F} 12$ with $10 \%$ FBS was added to inactivate trypsin. The cell suspension was filtered through a $40 \mu \mathrm{m}$ cell strainer and filtered cells were dropped on solidified 1\% Matrigel coated wells to form a monolayer. $100 \mu \mathrm{l}$ of culture medium was added on top of the cell layer, and $500 \mu \mathrm{l}$ of culture medium were added to the bottom. Monolayers were used for experiments after 24 hours. For M cell development, $200 \mathrm{ng} \mathrm{ml} \mathrm{m}^{-1}$ RankL was added in both top and bottom compartments.

\section{Transport of mammalian orthoreovirus across intestinal monolayers in a Transwell model system}

Purified virus was serially diluted and subjected to a modified plaque assay analysis. ${ }^{19,33}$ In brief L929 cells were seeded at $1.2 \times 10^{6}$ cells per $\mathrm{mL}$ (or per well) in 6-well plates and upon monolayer formation, the medium was removed and virus diluted in phosphate-buffered saline (PBS) (137 mM NaCl, $3 \mathrm{mM} \mathrm{KCl}, 8 \mathrm{mM} \mathrm{Na} \mathrm{HPO}_{4}, 1.5 \mathrm{mM} \mathrm{KH} \mathrm{PO}_{4}, \mathrm{pH}$ 7.4) with $2 \mathrm{mM} \mathrm{MgCl}$. Virus was incubated on monolayers for $1 \mathrm{~h}$ at 
room temperature, and then the bottom solution was collected at 2 hours, 4 hours, 6 hours, and 12 hours after the addition of virus to the top of the Transwell system. Then, $10 \mu \mathrm{l}$ of the culture medium from the bottom chamber was collected and overlaid with $2 \mathrm{ml}$ of 1\% Bacto Agar (Becton Dickinson), 1× Medium 199 (Life Technologies) supplemented with $2.2 \mathrm{~g} \mathrm{~L}^{-1}$ sodium bicarbonate (Fisher Scientific), and $12 \mu \mathrm{g} \mathrm{ml} \mathrm{m}^{-1}$ trypsin (Worthington Biochemical) solution. Dilutions were made for $10^{-3}, 10^{-4}, 10^{-5}$, and $10^{-6}$ by using the previously made solution. Once the overlay solidified, the 6-well plates were placed at $37{ }^{\circ} \mathrm{C}$ in $5 \% \mathrm{CO}_{2}$ and plaques were counted 2 days later. The means and standard deviations were determined from two biological replicates done in triplicate.

\section{Transport of AVNs across intestinal monolayers in a Transwell model system}

Rhodamine 6G (Sigma-Aldrich) (15 $\mu \mathrm{l}, 10 \mathrm{mM})$ was incubated with $1.5 \mathrm{ml}$ of $\mathrm{OD}=3.0 \mathrm{GNCs}$ and AVNs overnight by shaking at $4{ }^{\circ} \mathrm{C}$ to allow loading of $\mathrm{R} 6 \mathrm{G}$ into the nanocages via diffusion. R6G loaded nanocages were collected by centrifugation at $9000 \mathrm{~g}$. Supernatants were removed, and the nanocages were re-dispersed in $1 \mathrm{ml}$ of deionized water under sonication. Initial R6G fluorescence intensities were recorded, and $150 \mu \mathrm{l}$ of R6G-loaded AVNs or nanocages were added to each Transwell. At $0,0.5,1,2,3,4,8,12,24,36$, and 48 hours, $50 \mu \mathrm{l}$ was collected from the lower compartment of the Transwell setup, and R6G fluorescence intensities were recorded. Each experiment was conducted in triplicate.

\section{Author contributions}

T. T. fabricated GNCs, isolated $\sigma 1$ protein, assembled $\sigma 1$ protein and gold nanocages together to fabricate AVNs, and performed characterization of GNCs, $\sigma 1$ protein, and AVN transport on monolayers. Y. Q. prepared the ISC monolayer system and performed the characterization of $M$ cells in 3D and $2 \mathrm{D}$ systems. Y. Q. preformed MRV transport tests. T. T. and Y. Q. performed imaging and analysis of data and cowrote the paper. L. D. B. assisted in isolating $\sigma 1$ protein, MRV transporting tests, and imaging. M. W., C. L. M., Q. W. and C. Y. provided the laboratory and equipment and assisted in editing the paper. C. Y. and Q. W. provided the financial support for the research. C. L. M., Q. W. and C. Y. supervised the experiment design and execution and provided materials.

\section{Conflicts of interest}

There are no conflicts of interest to declare.

\section{Acknowledgements}

Finical support: Dr Wang is grateful for the support from Crohn's \& Colitis Foundation of America (CCFA) Career Award
(No. 348137) and PhRMA Foundation Research Starter Award (No. RSGTMT17). Dr Yu would like to thank USDA-NIFA (grant no. 2016-07802) and USDA-ARS (award no. 019636-00001) for partially funding this research.

The authors thank D. Rollins for assistance with data analysis, K. Yoon for assistance in T1L $\sigma 1$ purification and qPCR, B. Bellaire and N. Peroutka-Bigus for assistance in immunofluorescence.

\section{References}

1 N. Lycke, Recent progress in mucosal vaccine development: Potential and limitations, Nat. Rev. Immunol., 2012, 12, 592-605.

2 E. L. Giudice and J. D. Campbell, Needle-free vaccine delivery, Adv. Drug Delivery Rev., 2006, 58(1), 68-89.

3 L. D. Bussiere, P. Choudhury, B. Bellaire and C. L. Miller, Characterization of a Replicating Mammalian Orthoreovirus with Tetracysteine-Tagged $\mu$ NS for Live-Cell Visualization of Viral Factories, J. Virol., 2017, 91(22), e01371-17.

4 J. L. Wolf, et al., Determinants of reovirus interaction with the intestinal $\mathrm{M}$ cells and absorptive cells of murine intestine, Gastroenterology, 1983, 85(2), 291-300.

5 M. Wang, Z. Gao, Z. Zhang, L. Pan and Y. Zhang, Roles of $M$ cells in infection and mucosal vaccines, Hum. Vaccines Immunother., 2014, 10, 3544-3551.

6 A. Helander, et al., The Viral 1 Protein and Glycoconjugates Containing 2-3-Linked Sialic Acid Are Involved in Type 1 Reovirus Adherence to M Cell Apical Surfaces, J. Virol., 2003, 77(14), 7964-7977.

7 P. W. K. Lee and G. Leone, Reovirus protein $\sigma 1$ : From cell attachment to protein oligomerization and folding mechanisms, BioEssays, 1994, 16(3), 199-206.

8 K. A. Knoop, et al., RANKL Is Necessary and Sufficient to Initiate Development of Antigen-Sampling $M$ Cells in the Intestinal Epithelium, J. Immunol., 2009, 183(9), 57385747.

9 T. Sato, et al., Single Lgr5 stem cells build crypt-villus structures in vitro without a mesenchymal niche, Nature, 2009, 459, 262-265.

10 G. Altay, et al., Self-organized intestinal epithelial monolayers in crypt and villus-like domains show effective barrier function, Sci. Rep., 2019, 9, 10140.

11 E. S. Barton, et al., Junction adhesion molecule is a receptor for reovirus, Cell, 2001, 104(3), 441-451.

12 T. Kanaya, et al., Development of intestinal $\mathbf{M}$ cells and follicle-associated epithelium is regulated by TRAF6mediated NF-кB signaling, J. Exp. Med., 2018, 215(2), 501519.

13 A. E. Gregory, R. Titball and D. Williamson, Vaccine delivery using nanoparticles, Front. Cell. Infect. Microbiol., 2013, 3, 13.

14 L. Zhao, et al., Nanoparticle vaccines, Vaccine, 2014, 32(3), 327-337. 
15 V. Pokharkar, et al., Gold nanoparticles as a potential carrier for transmucosal vaccine delivery, J. Biomed. Nanotechnol., 2011, 7(1), 57-59.

16 J. P. M. Almeida, E. R. Figueroa and R. A. Drezek, Gold nanoparticle mediated cancer immunotherapy, Nanomedicine, 2014, 10(3), 503-514.

17 S. E. Skrabalak, et al., Gold nanocages: Synthesis, properties, and applications, Acc. Chem. Res., 2008, 41(12), 1587-1595.

18 S. E. Skrabalak, L. Au, X. Li and Y. Xia, Facile synthesis of $\mathrm{Ag}$ nanocubes and Au nanocages, Nat. Protoc., 2007, 2, 2182-2190.

19 D. B. Furlong, M. L. Nibert and B. N. Fields, Sigma 1 protein of mammalian reoviruses extends from the surfaces of viral particles, J. Virol., 1988, 62(1), 246-256.

20 K. A. Dryden, et al., Internal structures containing transcriptase-related proteins in top component particles of mammalian orthoreovirus, Virology, 1998, 245(1), 33-46.

21 I. I. Mendez, L. L. Hermann, P. R. Hazelton and K. M. Coombs, A comparative analysis of Freon substitutes in the purification of reovirus and calicivirus, J. Virol. Methods, 2000, 90(1), 59-67.

22 M. J. E. Fischer, Amine coupling through EDC/NHS: a practical approach, in Surface Plasmon Resonance, Methods in Molecular Biology (Methods and Protocols), ed. N. Mol and M. Fischer, Humana press, 2010, vol. 627, pp. 55-73.

23 M. R. Ivanov, H. R. Bednar and A. J. Haes, Investigations of the mechanism of gold nanoparticle stability and surface functionalization in capillary electrophoresis, ACS Nano, 2009, 3(2), 386-394.

24 A. Fatehullah, S. H. Tan and N. Barker, Organoids as an in vitro model of human development and disease, Nat. Cell Biol., 2016, 18, 246-254.
25 W. de Lau, et al., Peyer's patch M cells derived from Lgr5(+) stem cells require SpiB and are induced by RankL in cultured 'miniguts', Mol. Cell. Biol., 2012, 32, 3639-3647.

26 J. D. Rouch, et al., Development of functional microfold (M) cells from intestinal stem cells in primary human enteroids, PLoS One, 2016, 11(1), e0148216.

27 Y. Yin and D. Zhou, Corrigendum: Organoid and Enteroid Modeling of Salmonella Infection, Front. Cell. Infect. Microbiol., 2018, 8, 257.

28 M. B. Wood, D. Rios and I. R. Williams, TNF- $\alpha$ augments RANKL-dependent intestinal $\mathrm{M}$ cell differentiation in enteroid cultures, Am. J. Physiol.: Cell Physiol., 2016, 311, C498C507.

29 H. Gehart and H. Clevers, Tales from the crypt: new insights into intestinal stem cells, Nat. Rev. Gastroenterol. Hepatol., 2019, 16, 19-34.

30 N. A. Mabbott, D. S. Donaldson, H. Ohno, I. R. Williams and A. Mahajan, Microfold (M) cells: Important immunosurveillance posts in the intestinal epithelium, Mucosal Immunol., 2013, 6, 666-677.

31 H. W. Virgin, R. Bassel-Duby, B. N. Fields and K. L. Tyler, Antibody protects against lethal infection with the neurally spreading reovirus type 3 (Dearing), J. Virol., 1988, 62(12), 4594-4604.

32 H. W. Virgin IV, M. A. Mann, B. N. Fields and K. L. Tyler, Monoclonal antibodies to reovirus reveal structure/function relationships between capsid proteins and genetics of susceptibility to antibody action, J. Virol., 1991, 65(12), 67726781.

33 J. K. Middleton, et al., Thermostability of Reovirus Disassembly Intermediates (ISVPs) Correlates with Genetic, Biochemical, and Thermodynamic Properties of Major Surface Protein 1, J. Virol., 2002, 76(3), 1051-1061. 